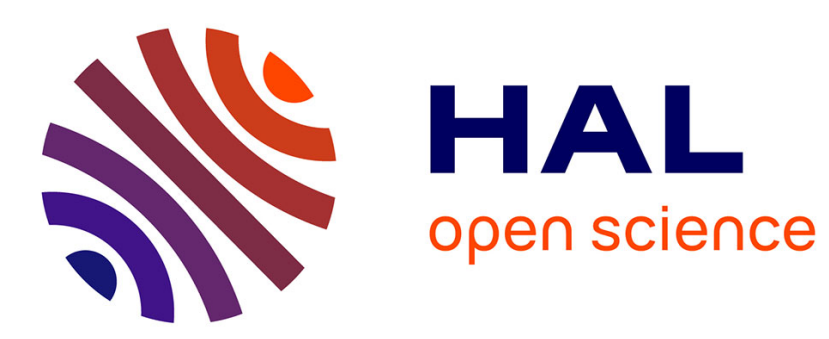

\title{
Que sait-on des trajectoires de pauvreté dans les pays riches?
}

Catherine Pollak, Bernard Gazier

\section{To cite this version:}

Catherine Pollak, Bernard Gazier. Que sait-on des trajectoires de pauvreté dans les pays riches?. Regards croisés sur l'économie, 2009, N4 "Pour en finir avec la pauvreté", pp.86-94. hal-00393492

\section{HAL Id: hal-00393492 \\ https://hal-paris1.archives-ouvertes.fr/hal-00393492}

Submitted on 9 Jun 2009

HAL is a multi-disciplinary open access archive for the deposit and dissemination of scientific research documents, whether they are published or not. The documents may come from teaching and research institutions in France or abroad, or from public or private research centers.
L'archive ouverte pluridisciplinaire HAL, est destinée au dépôt et à la diffusion de documents scientifiques de niveau recherche, publiés ou non, émanant des établissements d'enseignement et de recherche français ou étrangers, des laboratoires publics ou privés. 


\section{QUE SAIT-ON DES TRAJECTOIRES DE PAUVRETE DANS LES PAYS RICHES ? ${ }^{12}$}

Catherine Pollak, doctorante au CES - Matisse (université Paris 1). Sa thèse porte sur la protection sociale et les nouveaux risques sociaux en Europe. Bernard GAZIER, professeur à l'université Paris 1, chercheur au CES - Matisse. Il a notamment écrit en 2003 Tous « sublimes ». Vers un nouveau plein-emploi, aux éditions Flammarion.

Le développement des données longitudinales, principalement appuyé sur la technique statistique des «panels", a rendu possible depuis une trentaine d'années dans les pays industrialisés l'apparition d'un large éventail de recherches centrées sur la dynamique des phénomènes de pauvreté et d'exclusion sociale. Ces études de la pauvreté en termes de trajectoires abordent notamment les thèmes suivants: genre, structure familiale, emploi, logement, quartier, mais aussi pauvreté et santé, état psychiatrique, délinquance, migrations, mortalité, grossesses adolescentes, origine ethnique/couleur de peau, etc.

Le présent article part de quelques remarques sur l'intérêt de ce type de recherche et sur la situation française dans ce domaine (I) avant de résumer les principaux résultats de ces travaux (II). Une dernière section (III) discute brièvement de leurs conséquences en matière de compréhension générale de la pauvreté et de politiques publiques.

\section{Les études longitudinales de la pauvreté et le retard français}

L'étude longitudinale des trajectoires de pauvreté présente des avantages méthodologiques majeurs, puisqu'elle offre une image plus complète que celle des effets nets qui ressortent en coupe transversale. Elle permet de montrer l'hétérogénéité des expériences de pauvreté, en distinguant au sein d'un «groupe » de pauvres la part de la pauvreté transitoire et celle de la pauvreté récurrente ou chronique. Le suivi des mêmes individus sur plusieurs années, ce qui est la définition des enquêtes en termes de «panel », permet d'étudier l'impact de certains événements sur les transitions vécues par les individus, et d'en percevoir les effets différés ainsi que les conséquences à long terme. En dehors de l'effet des événements d'emploi (chômage, emploi précaire, etc.) ou familiaux (comme le divorce), il est possible de distinguer les rôles d'autres variables sur les trajectoires de pauvreté et la vulnérabilité de certains groupes, en fonction des caractéristiques individuelles, de l'environnement local, ou encore de l'évolution du contexte social et économique. Grâce à la connaissance de la chronologie des événements, plus fiable et plus précise que celle que l'on peut obtenir par les enquêtes

\footnotetext{
${ }^{1}$ Article paru en septembre 2008 dans Regards Croisés sur 1 Economie, $\mathrm{n}^{\circ} 4$ «Pour en finir avec la pauvreté », p.86-94.

${ }^{2}$ Cet article reprend et synthétise les principaux développements de :

POLLAK C. et GAZIER B., 2008, "L'apport des analyses longitudinales dans la connaissance des phénomènes de pauvreté et d'exclusion sociale : un survey de la littérature étrangère”, Les travaux de 1 Observatoire nationale de la pauvreté et de l'exclusion sociale 2007 - 2008, La documentation française, pp 561 - 604.
} 
rétrospectives, il est possible de reconstruire les trajectoires des personnes pauvres en identifiant la manière dont les événements apparaissent, s'enchaînent et se développent, et de mettre en évidence certaines relations de causalité.

Les études dynamiques de la pauvreté sont encore relativement peu développées en France. Les travaux pionniers remontent aux années 1980 et ont été menés, sous l'impulsion de J.C. Ray, à partir du «panel lorrain » qui offrait un suivi des personnes dont les revenus étaient proches du niveau du revenu minimum d'insertion (RMI). Depuis plusieurs études ont été réalisées (Michel Dollé, p.97 du même numéro).

Ce manque s'explique en partie par des difficultés conceptuelles et méthodologiques intrinsèques à l'analyse longitudinale, qui ont longtemps contribué à la préférence pour l'étude transversale de la pauvreté. Les données longitudinales apportent des informations extrêmement riches, mais qui ne sont pas évidentes à mobiliser (questions de coût, problèmes d'attrition ${ }^{3}$, d'erreurs de mesure, d'interprétation, etc.). Mais le rattrapage tardif de la littérature scientifique française, en comparaison avec d'autres pays industrialisés, provient surtout de la carence de données d'enquêtes de panel nationales disponibles en France avant la mise en place du panel européen en 1994 (European Community Household Panel ECHP -, devenu European Union Statistics on Income and Living Conditions - EU-SILC en 2004, et Statistiques sur les revenus et les conditions de vie - SRCV - en France).

La littérature étrangère sur données longitudinales a prouvé son intérêt. Si les constats établis à l'étranger peuvent servir de point de départ à la réflexion en France, les résultats de ces études ne sont pas forcément généralisables. Il est donc important que l'analyse dynamique se développe en France, afin que soient prises en compte les particularités du contexte institutionnel qui influencent les trajectoires des individus (protection sociale, droit du travail, montant des transferts, infrastructures, etc.). Cette approche est mobilisable et utile pour l'évaluation des politiques publiques, pour améliorer le ciblage des populations, pour éprouver les moyens de sécurisation des trajectoires afin de protéger des phénomènes de rechute et de récurrence de la pauvreté, pour évaluer l'efficacité des politiques sur les différents profils et types de pauvreté, et pour reconnaître le rôle des différentes institutions. Les initiatives récentes en France dans ce domaine de recherche sont donc à encourager et renforcer.

Enfin, les expériences étrangères ont montré l'intérêt de recourir à des approches pluridisciplinaires. Elles permettent d'améliorer les données longitudinales et leur potentiel d'exploitation (variété des domaines abordés, adaptation des variables aux méthodes d'analyse, qualité des réponses prospectives et rétrospectives obtenues, articulation des données qualitatives et quantitatives, etc.) ainsi que la réflexion méthodologique, l'interprétation des résultats et la formulation de recommandations. Le pragmatisme et la pluridisciplinarité invitent en définitive à mettre l'accent sur la complémentarité des différentes sources et outils d'analyse.

\section{Les principaux résultats disponibles}

Les études longitudinales apportent des éléments de connaissance sur les phénomènes de pauvreté, qui permettent d'apporter des réponses à trois grandes questions.

${ }^{3}$ On parle d'attrition lorsque des individus quittent un panel, ce qui empêche le chercheur de suivre leur évolution. 


\subsection{Quels sont les événements déclencheurs de la pauvreté, et y a-t-il des caractéristiques protectrices?}

Lorsque l'on dispose de données de suivi des individus sur au moins deux années, on peut déjà compléter l'analyse statique et «classique» de la pauvreté monétaire par des mesures dynamiques. On peut ainsi observer l'incidence de la pauvreté (part de la population qui traverse des épisodes de pauvreté), et analyser l'origine de ces épisodes de pauvreté (facteurs d'entrée et de sortie, caractéristiques des groupes «protégés » et des groupes «vulnérables »).

\section{"Aujourd'hui en Europe, selon les données longitudinales, environ un tiers de la population fait, sur cinq ans, l'expérience de la pauvreté."}

Depuis les premiers travaux menés aux États-Unis dans les années 1970, on constate un fort turnover dans la pauvreté d'une année à l'autre. Aujourd'hui en Europe, selon les données longitudinales (ECHP), environ un tiers de la population fait, sur cinq ans, l'expérience de la pauvreté $^{4}$ [Fouarge et Layte, 2005]. L'incidence de la pauvreté est donc bien plus élevée que le taux de pauvreté observé en coupe transversale (12\%), et elle augmente avec la durée de la période d'observation. La pauvreté est surtout transitoire et la majorité de ses épisodes sont de courte durée : les études montrent en général qu'un tiers des personnes qui se trouvent en dessous du seuil de pauvreté n'y sont plus l'année suivante. Le constat est le même dans les travaux qui s'appuient sur les fichiers administratifs des bénéficiaires de prestations sociales (en particulier l'Aid for Families With Dependent Children aux États-Unis, ou l'Income Support au Royaume-Uni).

L'apport principal de ces résultats est de mettre en évidence l'hétérogénéité des expériences de la pauvreté. Même s'ils montrent que la majorité des personnes qui en font l'expérience ne restent pas dans la pauvreté, il ne faut pas pour autant minimiser l'importance de la pauvreté chronique.

Les expériences de pauvreté sont hétérogènes en termes de durée et de sévérité. Afin de mieux comprendre les différences dans la vulnérabilité des individus face au risque de pauvreté, on peut identifier certaines caractéristiques qui, sans être des causes de pauvreté, lui sont associées. Au niveau européen, les caractéristiques les plus associées à un risque élevé de pauvreté sont une grande taille du ménage, le fait d'être sans emploi et d'avoir un niveau d'éducation faible. Un mauvais état de santé ou un âge élevé du chef de ménage apparaissent souvent comme des facteurs discriminants. Le facteur le plus protecteur est l'emploi, surtout lorsqu'il est stable, à temps plein et/ou que les deux membres du ménage travaillent. Un niveau d'éducation élevé protège également de l'entrée dans la pauvreté, mais n'en garantit pas la sortie [Fouarge, Layte, 2005, Smith, Middleton, 2007, Valletta, 2006].

Grâce au suivi en panel, on peut mettre en évidence certaines relations de causalité, et identifier les événements déclencheurs d'entrée et de sortie de la pauvreté. Les études insistent sur le rôle majeur des changements dans le statut d'emploi (perte d'emploi/retour à l'emploi à temps plein), dans les revenus (montant, source), et dans le statut marital (séparation/mise en couple). Le rôle de ces facteurs varie selon les pays, le contexte institutionnel et les traditions familiales (transferts sociaux, emploi des femmes, taille des ménages, etc.).

\footnotetext{
${ }^{4} \mathrm{Au}$ sens monétaire : revenus en dessous de $60 \%$ du revenu médian
} 


\subsection{Peut-on repérer une spirale de la pauvreté ?}

De plus en plus d'études complètent cette analyse de la pauvreté monétaire par des indicateurs de privations et de vulnérabilité dans l'analyse de moyen terme. Seule une petite partie des sorties de la «pauvreté monétaire»sont aussi des sorties de la «pauvreté en termes de conditions de vie », l'amélioration des privations étant plus lente que celle des revenus. Quant à l'entrée dans la pauvreté, elle se manifeste initialement par la dégradation d'un indicateur de «pauvreté subjective ${ }^{5}$ », les prestations sociales arrivant plutôt une fois le processus enclenché.

Les études permettent également d'apporter des éléments de connaissance sur la sévérité des épisodes de pauvreté, ou encore de vérifier l'hypothèse d'un cercle vicieux d'exclusion sociale. Gallie et al. [2003] ont ainsi mis en évidence une spirale descendante des désavantages, où le chômage augmente le risque de pauvreté, qui accroît à son tour les difficultés de retour à l'emploi et accentue l'isolement social.

La multiplicité et la variété des transitions observées rendent cependant difficile la mise en évidence de trajectoires types de pauvreté.

À long terme, on peut étudier en profondeur les trajectoires des ménages, et mettre en évidence les phénomènes de récurrence et de persistance des périodes de pauvreté. La pauvreté chronique, qui comprend ces deux dimensions, est loin d'être négligeable : on estime par exemple qu'elle concerne un tiers des pauvres en Allemagne et près de $40 \%$ aux ÉtatsUnis [Valletta, 2006]. La persistance de la pauvreté est particulièrement inquiétante chez les enfants dans les pays anglo-saxons [Bradbury et al., 2001].

Face aux débats sur la «culture de la dépendance », de plus en plus de travaux cherchent à identifier empiriquement les rôles respectifs des facteurs de «structure » (mutations économiques, institutions sociales, mouvement sociaux, etc.) et d' «agency» (arbitrages individuels) dans l'explication des trajectoires de pauvreté.

D'autres travaux récents cherchent à identifier les effets structurels dans les trajectoires, en tentant de distinguer les transitions structurelles (comme la chute dans la pauvreté liée à la perte de capitaux) des transitions stochastiques (comme l'entrée dans la pauvreté liée à la « malchance ») [Carter et Barrett, 2006].

\section{«Le facteur essentiel de sortie de la pauvreté est l'emploi. Mais l'importance de la pauvreté dans l'emploi montre que l'emploi à lui seul n'est pas une garantie contre la pauvreté, et que sa qualité (stabilité, niveau de salaire) doit faire partie intégrante des politiques publiques. »}

L'analyse sur le long terme permet de mettre en perspective les explications qui relèvent de la famille et de l'emploi en intégrant des variables de contexte. Des études britanniques et américaines montrent que ces facteurs (quartier, bassin d'emploi, accès aux services, etc.) jouent un rôle important même si leur puissance explicative est moins forte que les facteurs individuels.

La comparaison internationale est très instructive et souvent utilisée pour l'évaluation des politiques sociales et d'emploi, et l'étude des performances des pays selon leur régime d'Étatprovidence. L'influence du contexte institutionnel et des régimes d'État-providence est importante non seulement sur les taux de pauvreté, mais également sur la prévention de la

\footnotetext{
${ }^{5}$ Sur la distinction entre ces trois conceptions de la pauvreté, voir RCE pp
} 
pauvreté, sa fréquence et sa durée. On constate notamment une meilleure prévention de la pauvreté dans les pays dits sociaux-démocrates, une pauvreté plus fréquente et plus durable dans les pays libéraux et du Sud, les pays corporatistes-conservateurs occupant une position intermédiaire. Quant aux probabilités de sorties de la pauvreté, elles sont fortes, puis baissent rapidement dans ce dernier groupe de pays, alors que dans les pays libéraux et du Sud, elles sont plus modérées et plus constantes dans la durée.

\subsection{Quelles sont les conséquences à très long terme de la pauvreté dans l'enfance ?}

À très long terme, l'analyse porte sur les phénomènes de transmission et de reproduction intergénérationnelle. Ces travaux, qui nécessitent des données de suivi difficiles à rassembler (enquêtes longitudinales dépassant la décennie, suivis de cohortes complétés par des questions rétrospectives), ont surtout été menés au Royaume-Uni et aux États-Unis, avant de commencer à se développer dans d'autres pays industrialisés dans les années 1990.

Ils cherchent d'une part à déterminer la relation entre les handicaps que cumulent les enfants de ménages pauvres et leurs résultantes à l'âge adulte (souvent mesurées par des indicateurs de bien-être corrélés au revenu : niveau d'éducation, santé, réussite sur le marché du travail, etc.).

Les études montrent que l'héritage intergénérationnel des désavantages est important, et ce quelles que soient les mesures de bien-être mobilisées. Les conséquences de la pauvreté dans l'enfance ou à l'adolescence peuvent durer tout au long du cycle de vie, et se transmettre de génération en génération. Ces phénomènes de reproduction auraient même tendance à s'accentuer selon certaines études britanniques [Blanden et Gibbons, 2006]. Contrairement à une idée reçue, des comparaisons internationales montrent qu'il y a moins de mobilité professionnelle et des revenus entre les générations aux États-Unis et au Royaume-Uni qu'en Allemagne, au Canada, et dans les pays d'Europe du Nord [Corak, 2004].

La recherche des relations de causalité dans ces processus de transmission est cruciale, puisqu'elle renvoie directement à l'impact des politiques fiscales, sociales, de santé et d'éducation. Les principales études visant à expliquer la transmission de la pauvreté insistent sur le rôle primordial du contexte familial et les circonstances de la scolarité des enfants sur leur développement, alors que le manque de revenu lui-même est au second plan.

Les études visant à distinguer l'effet de la famille d'autres effets de l'environnement (quartier, communauté, aide sociale, etc.) se multiplient. Il en est de même pour les travaux portant sur les politiques publiques, qui montrent les effets contrastés de l'aide sociale sur les différents groupes de bénéficiaires. Cependant, la combinaison nécessairement complexe des facteurs qui jouent sur les trajectoires des enfants oblige à prendre des précautions avec leurs résultats.

\section{Quelques conséquences pour la compréhension de la pauvreté et pour les politiques publiques}

Les recherches longitudinales sur la pauvreté ont trouvé dans les théories existantes de la pauvreté et de l'exclusion des points d'appui pour s'orienter. En retour, leurs apports ont permis d'affiner ces théories. Une tradition de recherche insiste sur la responsabilité individuelle des pauvres, et l'autre sur les contraintes, souvent collectives, auxquels ils sont soumis.

Depuis la seconde moitié $\mathrm{du} \mathrm{XX}^{\mathrm{e}}$ siècle, la question des choix intertemporels est passée au premier plan de la première tradition. D'un côté alors, avec l'idée d'optimisation intertemporelle, la responsabilité des pauvres semble renforcée: certains pauvres 
minimiseraient rationnellement leurs efforts productifs et devraient donc être dissuadés de poursuivre dans ce qui risque de devenir un choix de vie. D'autres auteurs en revanche insistent sur l'imperfection de certains marchés (tels que la formation professionnelle) et sur l'extrême difficulté pour les pauvres à sortir des contraintes de court terme qui les empêchent d'optimiser quoi que ce soit. Le point de consensus porte sur les enfants, clairement irresponsables, et nécessitant des interventions vigoureuses afin d'être engagés tôt dans la bonne direction.

Symétriquement, mais avec moins de controverses, les courants qui se centrent sur les contraintes collectives ont développé une ample réflexion sur les conditions rendant possibles des choix intertemporels. Dès lors, l'étude des trajectoires des pauvres rejoint les travaux de la sociologie de la domination, introduisant par exemple les déficits de capital scolaire et symbolique dans des champs polarisés, étudiant aussi les processus d'intériorisation qui conduisent les pauvres à se satisfaire de leur position.

L'approche longitudinale permet de pondérer les deux classes de déterminants, individuels et collectifs. La distinction entre pauvreté transitoire, récurrente et persistante permet de sortir d'une vision uniformisante d'une pauvreté stable. Certaines périodes (chômage, naissance d'un enfant, maladie...) peuvent être des occasions soudaines d'entrée dans la pauvreté. Pour protéger les personnes lors de ces transitions qui peuvent s'avérer «critiques », il convient de gérer les risques plus élevés de pauvreté chez certains groupes (personnes âgées, femmes, jeunes, minorités ethniques, etc.) et à certains moments de la vie (entrée dans la vie active, naissances d'enfants, séparations, etc.). Les enquêtes confirment que le facteur essentiel de sortie de la pauvreté est l'emploi. Mais l'importance de la pauvreté dans l'emploi montre que l'emploi à lui seul n'est pas une garantie contre la pauvreté, et que sa qualité (stabilité, niveau de salaire) doit faire partie intégrante des politiques publiques. L'analyse longitudinale enfin permet de tester l'idée répandue de « dépendance à l'aide sociale », nourrie par l'image d'un groupe de pauvres figé véhiculée par les taux de pauvreté statiques : les éléments empiriques l'infirment. L'analyse longitudinale invite au contraire à offrir un revenu adéquat à ceux qui connaissent une pauvreté durable, puisque ces personnes souffrent d'encore plus de privations qu'on ne pouvait le penser - par conséquent, des sorties temporaires de la pauvreté ne peuvent suffire à les aider. Alors que les politiques se focalisent souvent uniquement sur la question des sorties de la pauvreté, l'approche dynamique, et c'est son intérêt majeur, permet d'évaluer la durabilité de ces sorties, et la réelle efficacité des mesures mises en place. 


\section{Bibliographie}

BLANDEN J., GIBBONS S., 2006, The Persistence of Poverty Across Generations. A View from two British Cohorts, Joseph Rowntree Foundation, The Policy Press, London School of Economics and Political Science.

BRADBURY B., JENKINS S.P., MICKLEWRIGHT J. (eds), 2001, The Dynamics of Child Poverty in Industrialised Countries, Cambridge, Cambridge University Press.

CARTER M.R., BARRETT C.B., 2006, « The economics of poverty traps and persistent poverty : an asset-based approach », Journal of Development Studies, vol. 42, ${ }^{\circ}$ 2, February.

CORAK M. (ed.), 2004, Generational Income Mobility in North America and Europe, Cambridge, Cambridge University Press.

FOUARGE D., LAYTE R., 2005, «Welfare regimes and poverty dynamics. The duration and recurrence of poverty spells in Europe », Journal of Social Policy, $\mathrm{n}^{\circ} 34$, issue 3, p. 407-426, Cambridge University Press.

GALLIE D., PAUGAM S., JACOBS S., 2003, « Unemployment, poverty and social isolation. Is there a vicious circle of social exclusion? », European Societies, 5(1), p. 1-32.

SMITH N., MIDDLETON S., 2007, «A review of poverty dynamics research in the UK », Report, Joseph Rowntree Foundation.

VALLETTA R.G., 2006, « The ins and outs of poverty in advanced economies. Government policy and poverty dynamics in Canada, Germany, Great Britain, and the United States », Review of Income and Wealth, Series 52, $\mathrm{n}^{\circ} 2$, June. 
This document was created with Win2PDF available at http://www.daneprairie.com. The unregistered version of Win2PDF is for evaluation or non-commercial use only. 